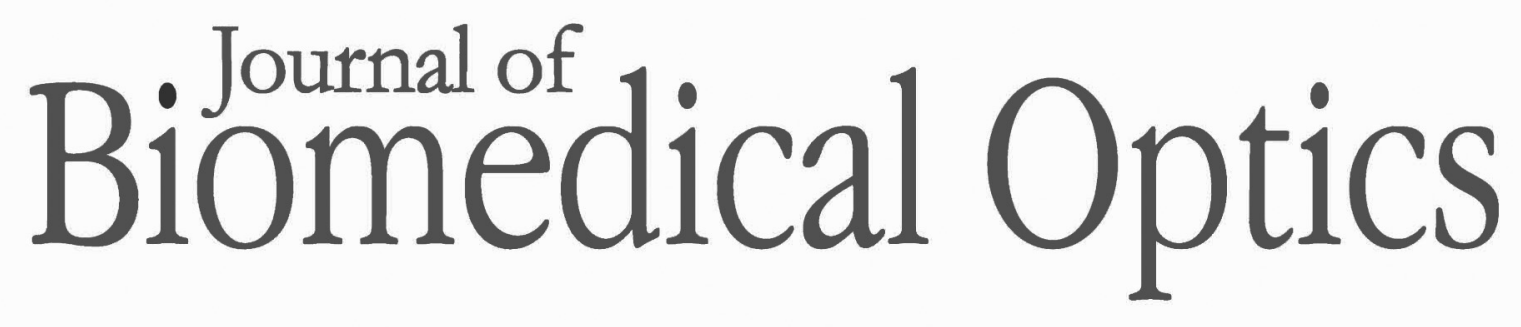

SPIEDigitalLibrary.org/jbo

\title{
Measuring incidence angle for through- the-objective total internal reflection fluorescence microscopy
}

Thomas P. Burghardt 


\title{
Measuring incidence angle for through-the-objective total internal reflection fluorescence microscopy
}

\author{
Thomas P. Burghardt \\ Mayo Clinic Rochester, Department of Biochemistry and Molecular Biology, and Department of Physiology and Biomedical Engineering, Rochester, \\ Minnesota 55905
}

\begin{abstract}
Total internal reflection fluorescence (TIRF) microscopy has the exciting laser beam incident beyond critical angle from the glass side of a glass/aqueous interface formed by the coverslip and aqueous sample. The aqueous side evanescent field decays exponentially with distance from the interface with penetration depth depending on incidence angle. Through-the-objective TIRF has the exciting laser focused at the back focal plane (BFP) creating a refracted parallel beam approaching the interface in the small gap between objective and coverslip, making incidence angle challenging to measure. Objective axial scanning does not affect incidence angle but translates beam and interface intersection detected by the fluorescence center of mass from fluorescent spheres attached to the aqueous side of the interface. Center of mass translation divided by the axial translation is the tangent of the incidence angle that is sampled repeatedly over objective trajectory to obtain a best estimate. Incidence angle is measured for progressively larger radial positions of the focused beam on the BFP. A through-theobjective TIRF microscope, utilizing a micrometer and relay lenses to position the focused beam at the BFP, is calibrated for incidence angle. Calibration depends on microscope characteristics and TIRF objective and is applicable to any interface or sample. $\odot 2012$ Society of Photo-Optical Instrumentation Engineers (SPIE). [DOI: 10.1117/1.JBO.17.12.126007]
\end{abstract}

Keywords: total internal reflection fluorescence; incidence angle; fluorescence microscopy; back focal plane; evanescent excitation profile.

Paper 12456 received Jul. 17, 2012; revised manuscript received Oct. 29, 2012; accepted for publication Nov. 1, 2012; published online Dec. 3, 2012.

\section{Introduction}

Illuminating volume shape and size in epi-illumination fluorescence microscopy is a critical parameter affecting resolution for scanning and widefield imaging methods. A smaller illumination volume defines the more resolved imaging capability. Nearfield excitation is nonpropagating and evanescent in one or more spatial dimensions. It is a widely used method with subdiffraction limit illumination volume. Total internal reflection fluorescence (TIRF) microscopy is a near-field method that has the exciting laser beam incident beyond critical angle, $\theta_{c}$, from the glass side of a glass/aqueous interface formed by the coverslip and aqueous sample. The evanescent field produced on the aqueous side decays exponentially with distance normal to the interface having a penetration depth that depends on incidence angle, excitation light wavelength, and refractive indices at the interface. ${ }^{1}$ Nominal depth is $\sim 100 \mathrm{~nm}$ for a glass/aqueous interface at visible wavelengths and a 66-deg incidence angle.

Through-the-objective TIRF uses a high numerical aperture (NA) objective to simultaneously refract incident light beyond critical angle incidence and collect fluorescence emission over a large solid angle. ${ }^{2}$ The high NA objective collects both propagating and evanescent emission due to the proximity of the TIR interface to the power-normalized emitting dipole. ${ }^{3}$ Evanescent emission intensity depends on the dipole-to-interface distance and is a substantial portion of the total emitted power for a dipole on the interface. Its collection enhances the signal-tonoise ratio critical to modern applications like single molecule

Address all correspondence to: Thomas P. Burghardt, Mayo Clinic Rochester Department of Biochemistry and Molecular Biology, and Department of Physiology and Biomedical Engineering, Rochester, Minnesota 55905; Tel: 507-2848120; Fax: 507-284-9349 (FAX); E-mail: burghardt@mayo.edu detection. Through-the-objective TIRF has the disadvantage that the exciting light impinges on glass elements internal to the objective, producing scattered and auto fluorescence light that tends to decrease signal-to-noise ratio. ${ }^{4}$

Prism-based TIRF utilizes a prism to couple the illuminating laser light to the TIR interface while sample fluorescence collection is through a water immersion objective. ${ }^{5}$ This method does not collect evanescent emission, but coupling the exciting laser beam to the sample via a prism external to the objective reduces contaminating background light compared to the through-the-objective TIRF method. Around-the-objective TIRF couples the exciting laser beam to the sample using an external prism and collects both propagating and evanescent emission, thereby combining positive features of prism based and through-the-objective TIRF. ${ }^{6}$

Through-the-objective TIRF is simple to implement, available as an option to most inverted research microscopes, and widely used. ${ }^{7,8}$ Principal features of implementation are depicted schematically in Fig. 1, where lens L1, focusing the exciting beam at the objective back focal plane (BFP), directs the laser to the lateral position on the BFP that sets incidence angle. Beam incidence angle determines penetration depth. ${ }^{1}$ Prism-dependent TIRF methods allow direct measurement of beam incidence angle because the illuminating laser beam is external to the microscope and user accessible. In through-the-objective TIRF, the illuminating laser makes the final approach to the totally reflecting interface in the small gap between the oil immersion objective and coverslip, making it practically inaccessible. I describe here a method to measure incidence angle in a throughthe-objective TIRF microscope.

0091-3286/2012/\$25.00 @ 2012 SPIE 


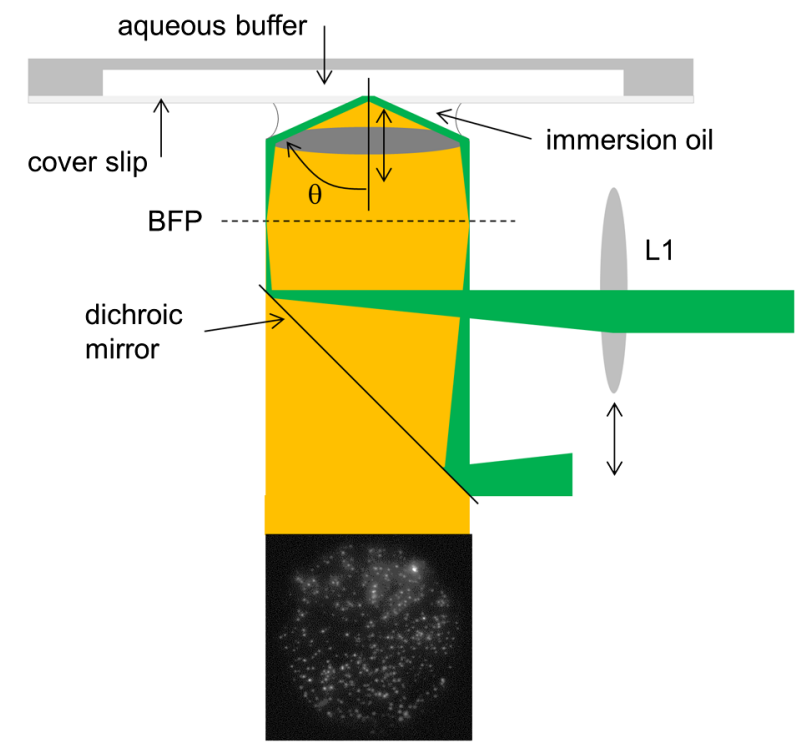

Fig. 1 Through-the-objective TIRF creates the evanescent field on the aqueous side of the glass/aqueous interface with the illuminating laser focused at the BFP then refracted by the objective at angles beyond $\theta_{c}$. Lens L1 displaces the focused spot of the BFP to adjust $\theta$. The objective also collects fluorescence from 40-nm-diameter spherical beads attached to the aqueous side of the coverslip. Fluorescence transmits the dichroic mirror and is formed into a real image by the tube lens onto the camera (tube lens and camera not shown) producing the image shown. Double-ended arrows indicate translating elements.

\section{Methods}

\subsection{Sample Preparation}

Red-orange carboxylate-modified 40-nm-diameter fluorescent spheres having excitation/emission maxima at 565/580 nm are from Molecular Probes (Life Technologies, Grand Island, New York). We diluted the stock $10^{4}$ fold into distilled water, giving sphere concentration of $1.4 \times 10^{11}$ spheres $/ \mathrm{mL}$. Experiments were conducted at room temperature.

A water-tight chamber was constructed from three coverslips. Two coverslips formed the top and bottom of the chamber, and the third was used to make $2 \times 30 \mathrm{~mm}$ spacers separating the top and bottom coverslips. The spacers were arranged along the long edges of the coverslips. The spacers and coverslips formed a $0.15-\mathrm{mm}$-thick rectangular solid volume with opposite ends open. Fluorescent spheres were flowed into one open end of the sample chamber and allowed to dry. Water was then flowed through the chamber, removing any loosely bound spheres. TIR illumination produced fluorescence from the cover-slip surface immobilized spheres.

\subsection{Microscopy}

Figure 2 shows the inverted microscope (Olympus IX71) with excitation and emission detection pathways. Double-edge arrows indicate translating elements, with the approximate spatial resolution and total travel (in brackets) indicated where appropriate. The 514.5-nm line from the argon ion laser (Innova 300, Coherent, Santa Clara, California) is linearly polarized by the Glan-Taylor $(\mathrm{P})$ polarizer. The polarization rotator $(\mathrm{PR})$ rotates linear light polarization to the desired orientation. The exciting light is shuttered at $\mathrm{S}$. The beam expander (BE) consists of a $4 \mathrm{X}$ microscope objective and a large-diameter

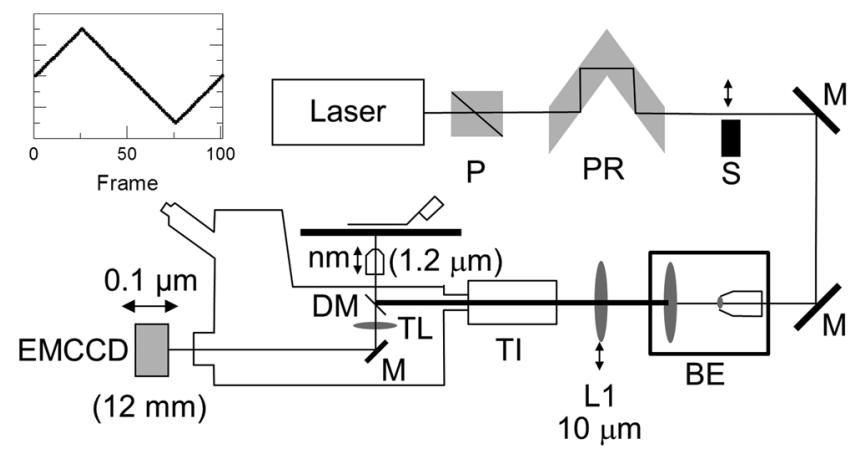

Fig. 2 Optical train for excitation and emission pathways serving the Olympus IX71 microscope. Double-edge arrows indicate translating elements with the approximate spatial resolution and total travel (in brackets) indicated where appropriate. The objective and camera axial displacement sawtooth pattern is indicated in the insert. Abbreviations are: Glan-Taylor polarizer $(\mathrm{P})$, polarization rotator $(\mathrm{PR})$, activatable shutter (S), mirror (M), beam expander (BE), lens (L1), TIRF illuminator $(\mathrm{TI})$, dichroic mirror (DM), and tube lens (TL).

long-focal-length $(250 \mathrm{~mm})$ lens. Exciting laser light is focused by L1 and then enters the TIRF illuminator (TI), reflects at the dichroic mirror (DM), and is focused on the sample by the objective. Alternatively, illumination is accomplished with an argon ion laser coupled directly to the TI through a fiber optic. This option is not shown in Fig. 2 but is described in detail below.

The objective (Olympus PlanApo 100X, 1.49 NA, $100 \mu \mathrm{m}$ working distance, and using Olympus immersion oil with refractive index 1.516 at $23^{\circ} \mathrm{C}$ ) translates along the optical axis under manual control using the microscope focus and with $\mathrm{nm}$ precision using a piezo nanopositioner (C-Focus, MCL). Emitted light is collected by the objective, transmitted by the dichroic mirror, then focused by the tube lens (TL) onto the camera (EMCCD, Hamamatsu C9100-13, Hamamatsu City, Japan). A microscope stage with leadscrew drives and stepper motors translate the EMCCD camera with submicron resolution (LEP, Hawthorne, New York). Computer control of the microscope is exercised through a custom written LabVIEW (National Instruments, Austin, Texas) routine and drivers supplied by the manufacturers.

Experiments were performed in two modes. In the moving objective/camera mode (mode 1), the objective and camera are scanned axially and synchronously over the sawtooth pattern shown in the Fig. 2 insert. The camera translates over a distance given by objective travel multiplied by objective axial magnification $\left(M^{2}\right.$ for $M$ the objective lateral magnification or $10^{4}$ for the $100 \times$ objective). The objective translates over $1.2 \mu \mathrm{m}$ with a step size of $24 \mathrm{~nm}$, implying the camera translates synchronously over $12 \mathrm{~mm}$ in steps of $240 \mu \mathrm{m}$. Synchronous movement of objective and camera keep the sample in focus (albeit with aberration) in the sequence of images taken over the scan. In the second mode (mode 2$)$, only the objective scans $(1.2 \mu \mathrm{m}$ total travel in steps of $24 \mathrm{~nm}$ ), hence the image sequence shows the sample in and out of focus depending on the frame. In both modes, the sample image sequence was aligned by computing the intensity overlap of each frame relative to the main frame at the center of the sawtooth curve (frame 51) as a function of relative two-dimensional frame translation (main frame remains fixed, no frame rotation). Optimized position maximizes overlap with the main frame. Experiments were performed in the two 
modes to address the practical concern that the image alignment algorithm could degrade when aligning in-focus with out-offocus images.

The TIRF illuminator affects the light path to the objective BFP, hence its impact on the beam displacement was assessed with geometrical optics. Illumination is accomplished with argon ion lasers coupled through a fiber optic or with the beam propagating in air directly from the output coupler of the laser. The former bypasses lens L1 and inputs directly to the TIRF illuminator. The latter (shown in Fig. 2) is coupled into the TIRF illuminator by L1. Figure 3 shows both pathways. The fiber optic output is a point source placed at the focal plane of L2 at the point intersected by the red line. This light propagates to the BFP and onto the sample plane through the objective along the red ray. L1 displaces the airborne beam from the optical axis of the TIRF illuminator. The beam follows the pathway indicated by the blue ray diagram. Fiber optic and airborne lasers have the same incidence angle. The evident divergence of blue and red rays causes a relative displacement of the TIR beams at the sample plane. We conducted experiments using either source and could not discern visually the relative displacement of the TIR beams. Displacement of the airborne beam by L1 translation using a micrometer, or identical displacement of the fiber optic point source by another micrometer, produced similar curves calibrating micrometer setting to incidence angle.

Figure 4 shows two parallel paths the incident TIR beam takes in the gap between objective and coverslip. The parallel beams leave the objective from two axial positions with the net displacement, $z_{j}-z_{51}$, indicated where subscripts $j$ and 51 refer to the $j$ 'th and 51 st frame. The 51 st frame is half way through the sawtooth pattern in Fig. 2. Objective axial displacement causes translation of the beam in the lateral plane indicated by $\vec{\rho}_{j}-\vec{\rho}_{51}$ for

$$
\left(z_{j}-z_{51}\right) \tan \theta=\left\{\begin{array}{ll}
-\left|\vec{\rho}_{j}-\vec{\rho}_{51}\right| & \text { for } z_{j}-z_{51}<0 \\
0 & \text { for } z_{j}=z_{51} \\
+\left|\vec{\rho}_{j}-\vec{\rho}_{51}\right| & \text { for } z_{j}-z_{51}>0
\end{array},\right.
$$

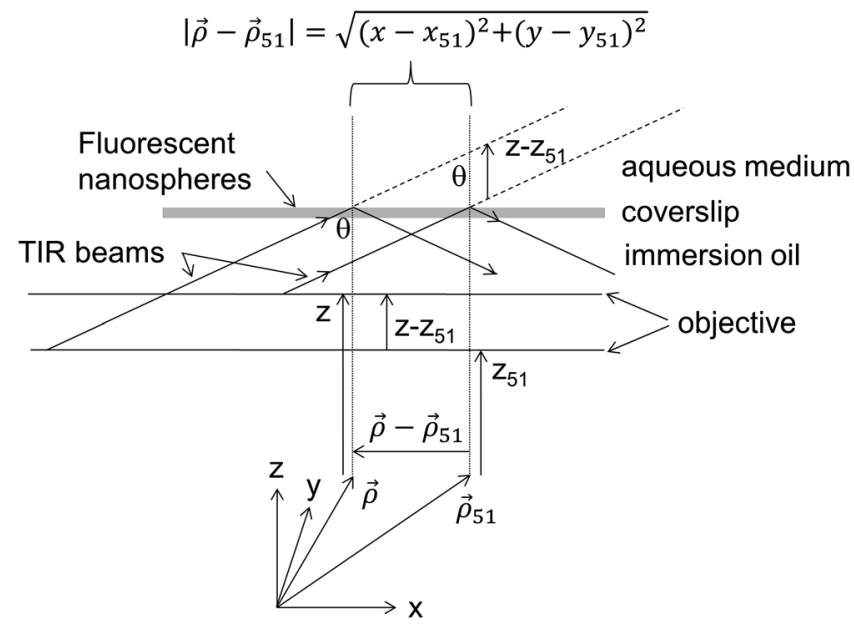

Fig. 4 Objective axial displacement, $z-z_{51}$, translates the beam intensity center of mass (COM) at the TIR interface through, $\vec{\rho}-\vec{\rho}_{51}$, on the aqueous side of the coverslip. Axial objective translation is expressed relative to its position in Frame 51 at the center of the axial saw-tooth trajectory shown in Fig. 2. Beam intensity COM is calculated using Eq. (2) and from the image of the fluorescent nanospheres collected by the objective.

where $\theta$ is the incidence angle and $\vec{\rho}_{j}$ the intensity center of mass (COM) in the sample plane in the $j$ 'th frame.

A $512 \times 512$ pixel intensity array, $c_{j}(i, k)$, where $i$ corresponds to pixels in the $y$-dimension and $k$ to pixels in the $x$ dimension relative to the camera, represents the fluorescent spheres image in frame $j$ under TIR illumination. The intensity COM,

$$
\vec{\rho}_{j}=\frac{p}{M} \frac{\sum_{i, k}\{i, k\} c_{j}(i, k)}{\sum_{i, k} c_{j}(i, k)}
$$

for $p=16 \mu \mathrm{m} /$ pixel, $M=$ objective magnification $=100$, and $\{i, k\}$ the position vector on the camera chip. Equation (2) is used to compute $\left|\vec{\rho}_{j}-\vec{\rho}_{51}\right|$ from $c_{j}(i, k)$ while $z_{j}-z_{51}$ is the readout from the piezo nanopositioner moving the objective.

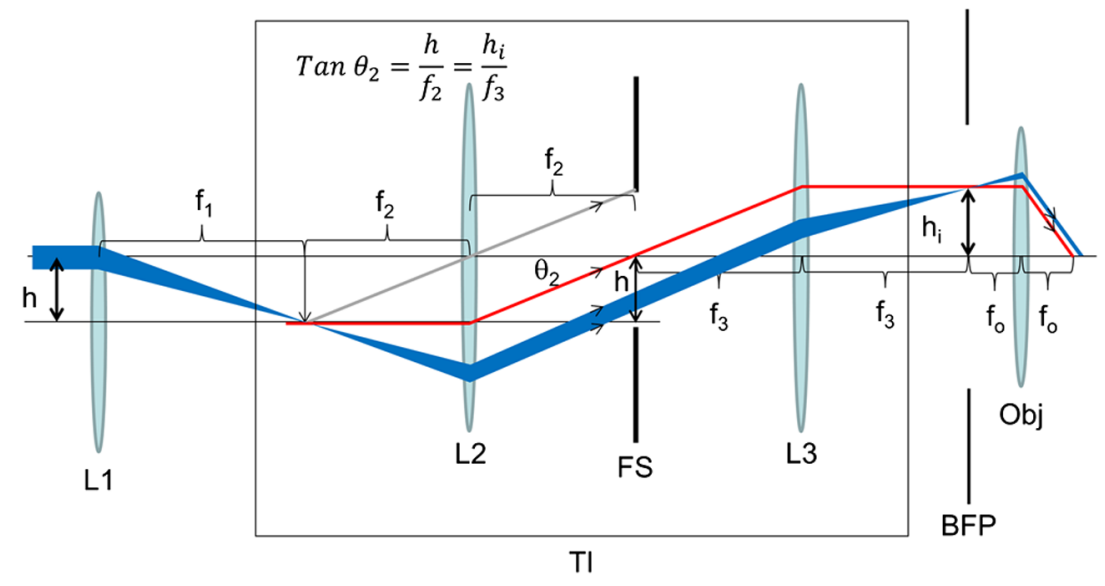

Fig. 3 Ray diagram for the Olympus TIRF illuminator (TI) and adjacent optical elements in the excitation beam. Illumination by the factory installed fiber optic bypasses lens L1 placing the point source of the fiber tip at the focal plane of L2 with light pathway indicated by the red line. The airborne laser is coupled to the TI via L1 with focal length $f_{1}$. L1 is displaced distance $h$ from the optical axis of the TIRF illuminator. The beam follows the pathway indicated in the blue ray diagram. L1 displacement, $h$, produces a focused beam at the BFP with height $h_{i}$ and magnification given by the ratio of focal lengths, $f_{3} / f_{2}$. The objective is shown with focal length $f_{o}$. Field stop, FS, controls the lateral size of the illuminated region at the focal plane of the objective. 


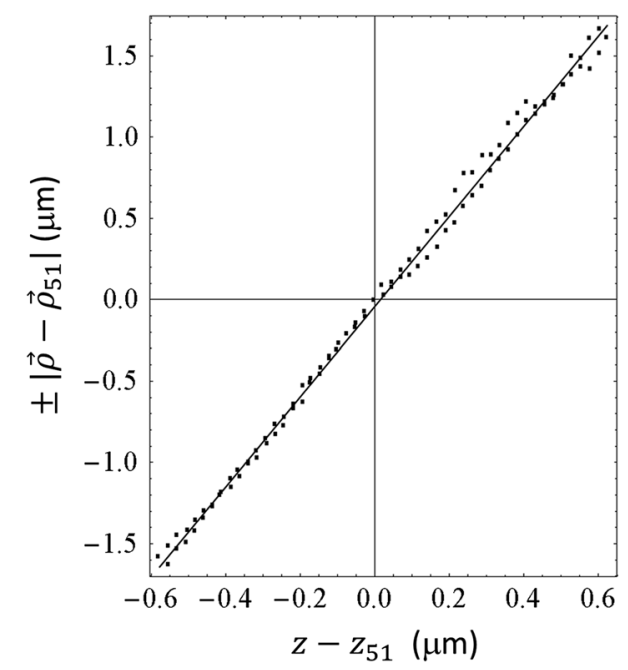

Fig. 5 The $\pm\left|\vec{\rho}_{j}-\vec{\rho}_{51}\right|$ versus $z_{j}-z_{51}$ plot for $1 \leq j \leq 100$ collected frames in a mode 2 scanning experiment. The line fitted to the curve has $\tan \theta$ for slope and incidence angle $\theta=70 \pm 0.1 \mathrm{deg}$.

Figure 5 indicates representative data for a mode 2 experiment with the $\pm\left|\vec{\rho}_{j}-\vec{\rho}_{51}\right|$ versus $z_{j}-z_{51}$ plot for $1 \leq j \leq 100$ collected frames. The fitted line to the curve has $\tan \theta$ for slope giving an incidence angle of $70.1 \pm 0.1 \mathrm{deg}$. Experiments performed in the two scanning modes address the practical concern that COM estimation could degrade for an out-offocus image.

\section{Results}

Figure 6(a) indicates incidence angle versus beam displacement in $\mathrm{mm}$ averaging data from six experiments using airborne illumination (three experiments) and fiber optic illumination (three experiments). Three experiments used the objective/camera synchronous translation mode (mode 1) and three experiments the objective only translation mode (mode 2 ). There was no evident performance difference related to the illumination method or scanning mode. Invariance under scanning modes 1 or 2 suggests the intensity COM is accurately approximated using Eq. (2) whether the image is in- or out-of-focus, and that the image alignment algorithm is adequate for alignment of an outof-focus image with its in-focus counterpart. Data in Fig. 6(a) also indicates the ratio of focal lengths for lenses L2 and L3 in the TI (Fig. 3), $f_{3} / f_{2} \approx 0.9$. Focal lengths $f_{2}$ and $f_{3}$ are several $\mathrm{cm}$ long.

Error bars indicate standard error of the mean for $n=6$. Beam displacement is the distance from the optical axis to the TIR beam where they intersect the BFP. The critical and maximum beam displacements are 1.58 and $1.77 \mathrm{~mm}$ corresponding to incidence angles of $61.6\left(\theta_{c}\right)$ and $79.4 \mathrm{deg}$, respectively. Experiments were performed by adjusting the illuminating source just past $\theta_{c}$ incidence with visual validation of TIR by observing the light streak in the interface plane at $\theta_{c}$ incidence completely disappear as we adjusted incidence beyond $\theta_{c}$. We see from Fig. 6(a) that this point is $\sim 4 \mathrm{deg}$ beyond $\theta_{c}$. At maximum beam displacement and beyond, emitted light intensity diminished as the illuminating beam exceeded the objective output aperture. The visual validation of TIR may not be as reliable a starting point for calibration as the maximum incidence judging by error estimates. It
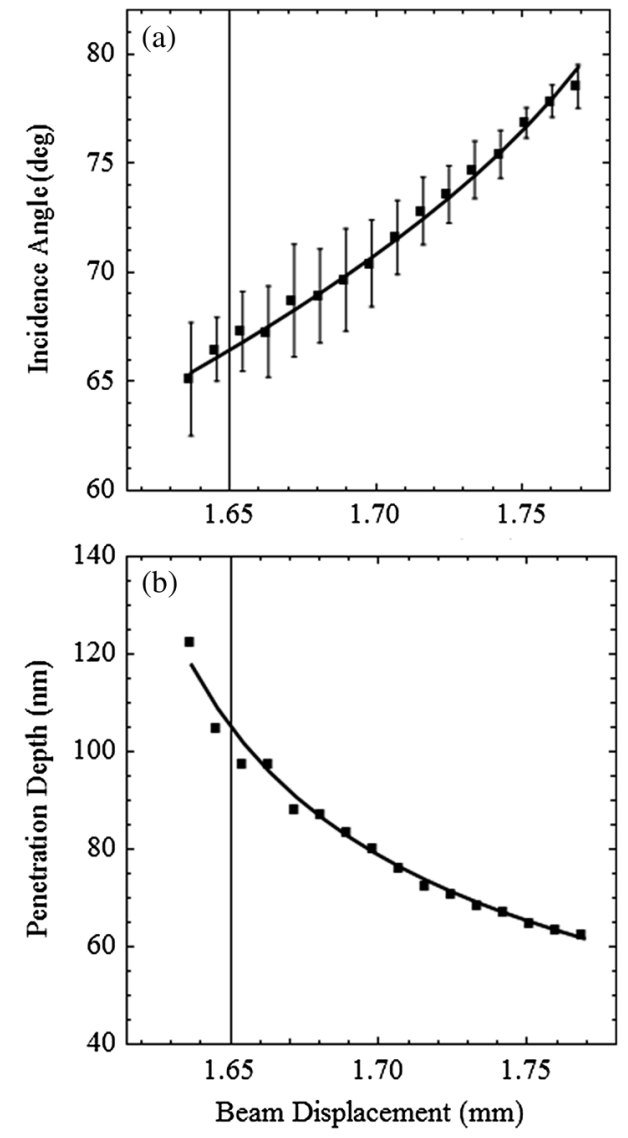

Fig. 6 (a) Incidence angle $(\theta)$ versus beam displacement in $\mathrm{mm}$ averaging data from six experiments using airborne illumination or fiber optic illumination. (b) Penetration depth of the evanescent field calculated using Eq. (3) and the incidence angle measured in A. Parameters used in Eq. (3) are: $\lambda=514.5 \mathrm{~nm}, n_{g}=1.516$, and $n_{w}=1.334$.

suggests that starting the calibration from maximum incidence may be better practice.

Figure 6(b) shows the calculated penetration depth,

$$
d=\frac{\lambda}{4 \pi} \frac{1}{\sqrt{n_{g}^{2} \sin ^{2} \theta-n_{w}^{2}}},
$$

for $\lambda$ the incidence light wavelength $(514.5 \mathrm{~nm})$ in air, $n_{g}$ and $n_{w}$ the refractive indices of glass and water (1.516 and 1.334), and $\theta$ from Fig. 6(a). ${ }^{1}$ It suggests penetration depth of $\sim 60 \mathrm{~nm}$ is achievable with the $100 \times 1.49$ NA Olympus TIRF objective.

\section{Discussion}

Illuminated volume minimization enhances emission source localization and background light rejection, both critically important in widefield microscopy. TIRF microscopy utilizes an evanescent field to selectively illuminate a sample within $\sim 100 \mathrm{~nm}$ of the TIR interface usually located at the coverslip-sample contact. Through-the-objective TIRF is widely used for this application due to its simple implementation on infinity-corrected optics and reliable performance. Atomic force microscopy combined with through-the-objective TIRF enabled the direct detection of the exponentially decaying evanescent field using a fluorescent marker. ${ }^{9,10}$ Mattheyses and Axelrod measured the evanescent field axial profile and penetration depth by imaging index- 
matched low refractive index spherical beads. ${ }^{4}$ They also showed that with through-the-objective TIRF, fractional contributions of evanescent and scattered light on the low refractive index side of the interface surface contributes $\sim 90 \%$ and $\sim 10 \%$ of the total intensity. The ratio of scattering versus evanescent intensity is probably smaller for prism-based TIRF. Both atomic force microscopy and spherical bead-imaging evanescent field calibration methods are elegant and practical; however, they must be reapplied to each situation in which basic optical characteristics of the TIR interface or sample change.

The approach described here measures the incidence angle of the illuminating beam. This characterization is independent of the optical characteristics of the TIR interface and sample in the evanescent field but depends mainly on objective NA and potentially other geometrical constraints related to the illuminating beam profile. In our case, the substitution of the airborne laser beam for the factory installed fiber optic coupling was shown not to affect incidence angle, hence it will not impact the evanescent field, although this conclusion could be surmised from the ray diagram in Fig. 3. Refractive indices of materials are often difficult to know absolutely because they depend on temperature, moisture content, and other parameters that all must be matched to the situation of interest. Additionally, biological matter is heterogeneous such that refractive index depends on spatial position in the sample. These factors will impact the evanescent field but not incidence angle.

A color-corrected TIRF objective should produce the same incidence angle for different illumination wavelengths, while penetration depth [Eq. (3)] will change as it depends explicitly and implicitly (from $n_{g}$ and $n_{w}$ ) on wavelength. However, lack of color corrected optics in the TIRF illuminator may yet impart wavelength dependence in the incidence angle calibration.

An absolute calibration of a TIRF objective could be stated as the incidence angle versus beam displacement, as in Fig. 6(a). This information is already coded in the objective NA because incidence angle and beam displacement are related by the sine condition (excluding Herschel condition obeying optics). Nonetheless, real TIRF instruments rely on micrometers and relay lenses to position the laser beam at the BFP. These devices, essentially the TIRF illuminator in Figs. 2 and 3, need calibration in the field. In this context, the incidence angle calibration is preferable to other methods because it depends only on microscope characteristics that do not change for a particular objective regardless of interface or sample optical characteristics. Hence, absolute calibration of the TIRF system should be easier, more accurate, and less expensive using incidence angle rather than evanescent field profile.

\section{Acknowledgments}

This work was supported by NIH grants R01AR049277 and R01HL095572 and by the Mayo Foundation.

\section{References}

1. M. Born and E. Wolf, "Basic properties of the electromagnetic field," in Principles of Optics, pp. 1-70, Pergamon Press, Oxford (1975).

2. A. L. Stout and D. Axelrod, "Evanescent field excitation of fluorescence by epi-illumination microscopy," Appl. Opt. 28(24), 5237-5242 (1989).

3. E. H. Hellen and D. Axelrod, "Fluorescence emission at dielectric and metal-film interfaces," J. Opt. Soc. Am B 4(3), 337-350 (1987).

4. A. Mattheyses and D. Axelrod, "Direct measurement of the evanescent field profile produced by objective-based total internal reflection fluorescence," J. Biomed. Opt. 11(1), 014006 (2006).

5. D. Axelrod, "Cell-substrate contacts illuminated by total internal reflection fluorescence," J. Cell Biol. 89(1), 141-145 (1981).

6. T. P. Burghardt, A. P. Hipp, and K. Ajtai, "Around-the-objective total internal reflection fluorescence microscopy," Appl. Opt. 48(32), 6120-6131 (2009).

7. D. Axelrod, "Total internal reflection fluorescence microscopy in cell biology," Traffic 2(11), 764-774 (2001).

8. D. Axelrod and G. M. Omann, "Combinatorial microscopy," Nat. Rev. Mol. Cell Biol. 7(12), 944-952 (2006).

9. A. Sarkar, R. B. Robertson, and J. M. Fernandez, "Simultaneous atomic force microscope and fluorescence measurements of protein unfolding using a calibrated evanescent wave," Proc. Natl. Acad. Sci. 101(35), 12882-12886 (2004).

10. M. S. Kellermayer et al., "Spatially and temporally synchronized atomic force and total internal reflection fluorescence microscopy for imaging and manipulating cells and biomolecules," Biophys. J. 91(7), 2665-2677 (2006). 